\title{
Utilisation of Sardinella longiceps in a Value Added Product Development in the Form of Omega-3 Enriched Instant Soup Powder
}

\author{
A.H.G.S. Udari ${ }^{1 *}$, I. Wickramasinghe ${ }^{1}$, M.V.E. Attygalle ${ }^{2}$ \\ ${ }^{I}$ Department of Food Science and Technology, University of Sri Jayewardenapura, Sri Lanka \\ ${ }^{2}$ Department of Zoology, University of Sri Jayewardenapura, Nugegoda, Sri Lanka \\ *shanika.udari@gmail.com
}

\begin{abstract}
Indian Oil Sardine or Sardinella longiceps is one of the most abundantly caught clupeoidsunder coastal pelagic fisheries in Sri Lanka. So far, there is no evidence of utilising it in a possible value added food product efficiently even though it is available throughout the year and easily affordable at a low cost.

Most of the small pelagic fish like Sardinella longiceps are considered as best sources of Omega-3 polyunsaturated fatty acids. Yet, there are no studies have been done to assure whether these health friendly fatty acids are consumed as they are, when fish is cooked. This study was designed to determine the effects of cooking on Omega-3 polyunsaturated fatty acids of Sardinella longiceps by comparing Gas chromatographic-mass spectroscopic fatty acid profiles of raw, boiled and fried fish and then utilising the fish in an innovative and value added product development emphasising the enhancement of Omega-3 PUFA intake. Omega-3 PUFA content of raw fish was $21.54 \%$ (of total fatty acids). With cooking, it has decreased and the resulted values were $14.23 \%$ and $2.83 \%$, respectively in boiled and fried fish. Since average per capita fish consumption of Sri Lanka is $30.5 \mathrm{~g}$, frying, which can be identified as the most popular cooking method of Sardines like small fish (due to lots of tiny bones present with lesser flesh), cannot provide the recommended minimum"EPA" Omega 3 intake (0.22 g per person) when taken alone without any other Omega-3 sources. As a solution for this issue, an instant soup powder which was incorporated with fish oil and fish powder derived from Sardinella longiceps was developed containing 9.31\% of Omega-3 PUFA, which is significantly higher than that of fried fish. It can provide $0.6 \mathrm{~g}$ of EPA and $1.9 \mathrm{~g}$ of DHA per serving which are higher than the recommended values of minimum daily intake. As a whole or a part of a meal, an instant soup enriched with Omega-3 can be more nutritious and a convenient way to elevate the Omega-3 intake.
\end{abstract}

Keywords: Omega-3 PUFA, EPA, DHA, Soup, Recommended daily intake 